\title{
Designer Compartment for Artificial Metalloenzymes through Controlled Liquid-Liquid Phase Separation
}

Tong Wu, Xianhui Chen, Guopu Huang, Yingjiao Deng, Anming Yang, Xinxin Feng and Yugang Bai*

State Key Laboratory of Chem-/Bio-Sensing and Chemometrics, and School of Chemistry and Chemical Engineering, Hunan University, Changsha, Hunan 410082, China

\begin{abstract}
Artificial metalloenzymes with different protein scaffolds, cofactors and functions have been prepared to expand the natural enzymatic repertoire with abiotic reactions. ${ }^{1-3}$ However, due to the sensitivity of metal centers toward various biomolecules, especially glutathione, ${ }^{4}$ low activity and turnover of artificial metalloenzymes in vivo are systematic problems not fully solved. Apart from straightforward routes such as the use of neutralizing agents, metal cofactors modification and directed evolution, one may notice that nature can create isolated microenvironments for diverse biological processes within cells. ${ }^{5}$ Following this way, here we report the in vivo assembly of artificial metalloenzymes based on HaloTag-SNAPTag fusion protein. These metalloenzymes have metal cofactors bound on protein interfaces, and can trigger liquid-liquid phase separation ${ }^{6,7}$ to form liquid condensates inside Escherichia coli. These condensates serve as membraneless, isolated compartments ${ }^{8}$ for artificial metalloenzymes to efficiently perform intracellular catalysis, mediating abiotic unmasking, coupling and polymerization reactions. The cellular compartmentalization also enables spatial control of reactions, either facilitating a cascade reaction within the confined spaces, or concurrent reactions with spatial separation. Such engineered Escherichia coli can work as whole-cell catalyst with confined metal species, colonizing at mice intestine to effect in vivo abiotic transformations with a lower chance of heavy metal poisoning. These results represent a systematic strategy to stabilize and potentiate artificial metalloenzyme in vivo, with potential applications in fields such as non-natural metabolism, fermentation and drug delivery.
\end{abstract}

\section{Introduction}

Artificial metalloenzymes (ArMs) are semi-synthetic catalysts resulted from the repurposing of natural metalloenzymes ${ }^{9}, 10$ or the incorporation of abiotic metal cofactors into protein scaffolds. ${ }^{11-15}$ With a bright vista of serving as powerful tools in expanding the naturally evolved reaction repertoire in cells, ${ }^{16} \mathrm{ArMs}$ ' development and application is significantly limited by the difficulty in installing of abiotic metal cofactors in vitro and in vivo, as well as the instability of the active metal centers in biological environment with various coordinative molecules present. Although the installation of metal cofactors can be simplified by choosing a high-affinity inhibitor or substrate for guided anchoring within a host protein, ${ }^{11-13,17,18}$ utilization of such approach may not grant enough stability for resulting ArMs with sensitive cofactors, even with the aid of directed evolution of the corresponding protein scaffold. The only solution for such scenarios, by far, is to "pin" the ArMs in an auspicious environment, such as the periplasm of Escherichia coli (E. coli), ${ }^{19-21}$ so that impact from surrounding "toxic" biomolecules can be minimized. 
One can easily see that the above strategy simply exploits bacterial periplasm as a reaction compartment, similar cases of which are not uncommon in nature. ${ }^{5}$ Enzymes are usually localized in respective microenvironment, such as an organelle, to avoid unnecessary interference with other molecules and biological processes. Thus, we wonder if artificial compartments can be established in cells specifically for desired abiotic catalysis, so that the strategy is not limited to the application in highly specific, naturally occurring spaces. Establishment of such compartments may even enable cascade reactions and spatially separated concurrent catalysis, which can be essential considerations for chemical biologists striving to create complex abiotic systems in living host cells. For these purposes, we noted that artificial membraneless organelles and compartments from liquid-liquid phase separation (LLPS) of biomacromolecules are emerging paradigms of cellular organization. ${ }^{6,822-24}$ As compartmentalization is commonly utilized by cells for positioning their cellular materials and controlling their metabolic processes, LLPSbased, concentrated proteins or nucleic acids can play vital roles in biosynthesis and cell metabolism, providing relatively isolated spaces for the active catalytic centers, yet allowing exchange of materials with the surrounding milieu due to their liquid-like nature. Therefore, one may straightforwardly expect the LLPS of ArMs to create compartments for a catalytic purpose. The key point for such design would be the creation of ArM's LLPS that can be reliably triggered, which is totally unprecedented.

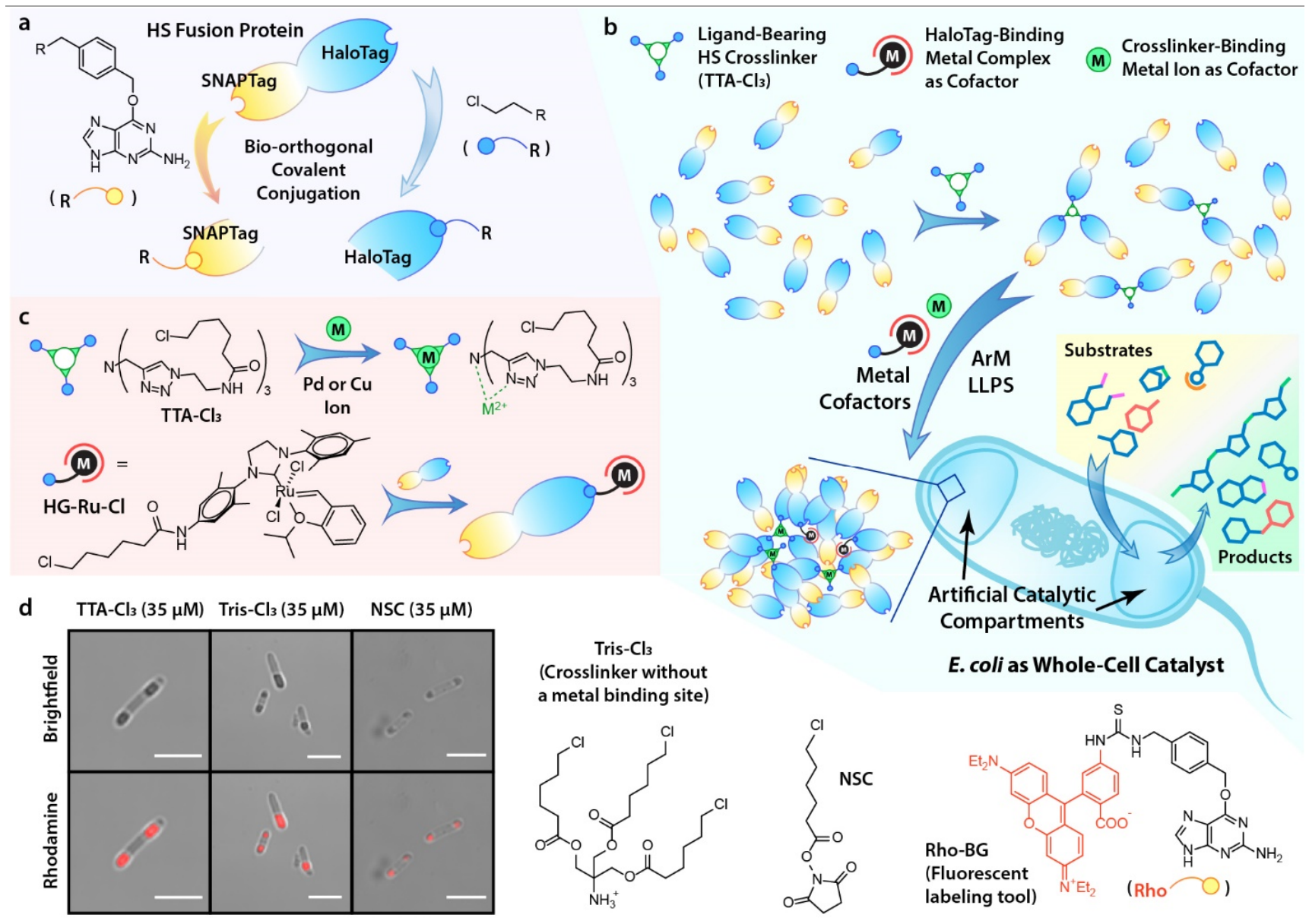


Figure 1. Illustrations of the creation of LLPS-based artificial catalysis compartments composed of artificial metalloenzymes with interficially bound metal cofactors within E. coli. (a) Illustration of HaloTag and SNAPTag mediated bio-orthogonal conjugation used for crosslinking and labeling in this study. (b) Overall schematic illustration of compartment creation through crosslinking-initiated LLPS process. (c) Cartoon schemes for the two modes for metal cofactor incorporation, using free metal ions or functionalized metal complexes with inherent catalytic ability. (d) Structures of cross-linkers that can induce the formation of LLPS compartments in HS-E. coli, and the images of such compartments. A rhodamine derivative that reacts with SNAPTag (Rho-BG) was used for fluorescent labeling of the compartments.

To create an ArM that can possibly undergo triggered LLPS, we set our attention on HaloTag, a self-labeling protein (SLP) tag that can covalently and bio-orthogonally accommodate terminal alkyl chlorides (Figure 1a and Supplemental Figure 1). This feature makes HaloTag equivalent to the widely utilized Streptavidin, based on which the first report on ArM assembly in a whole-cell set-up and its subsequent use as a catalyst in an in cellulo abiotic transformation was made possible. In fact, while we are working on this manuscript, Ward and Liang showed the capability of HaloTag serving as an ArM scaffold in vitro. ${ }^{13}$ More interestingly and importantly, HaloTag has a mediocre folding energy $\left(\Delta G_{\text {Folding }}=-5 \mathrm{kcal} / \mathrm{mol}\right),{ }^{25}$ thus it can undergo misfolding, which may create intrinsically disordered regions (IDRs) that promote aggregation and LLPS ${ }^{26}$ through multivalent IDR-IDR interactions. ${ }^{27}$ This aggregation tendency is also reflected by the report of a HaloTag mutant, named AgHalo, with a $\Delta G_{\text {Folding }}=-2$ $\mathrm{kcal} / \mathrm{mol}$, which was already employed as a fluorogenic tagging tool to detect protein aggregation in eukaryotes. $^{25,28,29}$ It was also reported very recently that LLPS-based membraneless compartments could be formed in prokaryotic E. coli using overexpressed, intrinsically disordered proteins. ${ }^{8}$ Thus, we considered HaloTag a nice candidate for serving as the protein scaffold for ArMs that can undergo LLPS to create catalytic compartments in E. coli, a well-established system simple and versatile enough for initial attempts.

\section{Results and Discussion}

To validate the above design, we constructed E. coli that could express HaloTag-SNAPTag (HS) fusion protein (HS-E. coli, Figure 1a). We chose HS protein instead of simple HaloTag for two reasons. First, similar to HaloTag, SNAPTag is also a SLP tag used as a protein aggregation detector, ${ }^{30}$ and may synergize with HaloTag. Fusion of the two is likely to promote their aggregation through mutual destabilization. Second, SNAPTag provides an additional and orthogonal conjugation site for metal cofactor installation and fluorescent labeling. We expect that a large amount of intracellular HS can show self-amplified aggregation and create artificial compartments with a trigger, which can be an artificial "crowding core" through controlled protein crosslinking (Figure 1b). The basis of such trigger design was analogous to RNA-promoted LLPS formation from RNA-binding proteins with IDRs. ${ }^{26}$ With a triggered initial aggregation, LLPS takes place like an overly concentrated solution seeded with a crystal.

For the trigger, we synthesized a tris(triazole) crosslinker, TTA- $\mathbf{C l}_{\mathbf{3}}$ (Figure 1b, 1c), which could simultaneously serve as a ligand for $\mathrm{Cu}^{\mathrm{I}}$ and $\mathrm{Pd}^{\mathrm{II}}$ while linking multiple $\mathrm{HS}$ protein units, causing an artificial "crowding core" that 
could serve as the trigger for macroscopic HS aggregation. If successful, the dimerization or trimerization process would also create a metal binding site on protein-protein interface (PPI). Working cofactor on PPI was already pioneered, ${ }^{10}$ and such design evaded the challenge in placing metal cofactors deep in a cavity but still maintained protective effect from macromolecular scaffolds. Indeed, by labeling the phase separated regions with $O^{6}$ benzylguanine-functionalized rhodamine (Rho-BG), it could be observed that the treatment of TTA-Cl 3 at HS- $E$. coli resulted in LLPS in as short as $1 \mathrm{~h}$ (Figure 1d and Supplemental Figure 2), with the aggregated phase growing larger over time (Supplemental Figure 2b, 2c). Other reagents that could link multiple HS proteins, such as $N$ succinimidyl chlorohexanoate (NSC) and $\mathbf{T r i s}-\mathbf{C l}_{3}$, were able to trigger LLPS, too, despite that they could not simultaneously provide metal binding sites (Figure 1d). The LLPS phenomenon was observed to be inheritable and, to some extent, reversible (Supplemental Figure 3a, 3b), consistent with the proposed mechanism of formation, i.e., strong protein-protein interactions through secondary bonds in a crowding environment. Besides, there was no significant difference in bacteria growth rate for those E. coli with or without LLPS regions (Supplemental Figure 3c). In vitro experiments using purified HS protein and TTA-Cl $\mathbf{C}_{3}$ also demonstrated successful formation of TTA$\mathbf{H S}_{2}$ and TTA-HS 3 , i.e., the "crowding cores", from the protein and the crosslinker, meanwhile a significant amount of TTA-HS $\mathbf{S}_{2}$ were detected in HS-E. coli containing LLPS droplets using gel analysis (Supplemental Figure 4a). Furthermore, the LLPS phenomenon could be reproduced in vitro, using concentrated HS protein in dextran-70 solution, a medium that simulates the intracellular physiological crowding environments ${ }^{8,31}$ (Supplemental Figure 4b). As a preliminary evaluation, purified TTA-HS $\mathbf{S}_{\mathbf{2}}$ and TTA-HS $\mathbf{S}_{\mathbf{3}}$ were tested in in vitro fluorogenic catalysis assays with supplemented $\mathrm{Cu}^{\mathrm{I}}$, and both showed better resistance toward malignant environment than monomeric TTA-HS, with the trimer outperforming the less hindered dimer (Supplemental Figure 5). Clearly, the cofactor-onPPI strategy was effective, and installation of the cofactor could simultaneously lead to desired, LLPS-based compartmentation that further stabilizes the created ArMs.

We next evaluated the catalytic efficiency of $\mathrm{Cu}^{\mathrm{I}}$-HS-ArMs in the artificial compartments created upon cofactormediated crosslinking and metal incorporation. Utilizing the widely adopted fluorogenic reaction between azidocoumarin and ethynylanisole, we could use confocal microscopic imaging to directly observe the coppermediated alkyne-azide cycloaddition (CuAAC). The result was highly encouraging: at E. coli-friendly copper concentrations of $10 \mathrm{nM}$ to $50 \mu \mathrm{M}$, strong fluorescence from the CuAAC product could be observed in the LLPS compartments in HS-E. coli in 0.5-6 h in different media (Figure 2a, row 1, and Supplemental Figure 6a, 6b). To the best of our knowledge, this result represents the best performance of intracellular CuAAC catalyst reported. Staining with acridine orange (AO) visualized bacterial nucleoid, whose fluorescence was totally separated from the ArM compartments, suggesting that the LLPS process did not involve E. coli's genomic DNA. Also, control groups using wildtype E. coli strain or HS-E. coli without IPTG showed no fluorescence for the abiotic catalysis, indicating the necessity of ArM compartments as the free copper complex, Cu-TTA-Cl $\mathbf{H}_{3}$, could be easily quenched in the cytoplasm (Figure 2a, column 2/6). By simply changing the metal on the PPI from $\mathrm{Cu}^{\mathrm{I}}$ to $\mathrm{Pd}^{\mathrm{II}}$, unmasking of propargyloxycarbonyl (Proc) protected rhodamine, another widely used fluorogenic model reaction, could be 
effected (Figure 2a, row 2 and Supplemental Figure 6c). Similarly, the fluorescent product from the reaction was co-localizing with the LLPS compartments, suggesting that $\mathrm{Pd}^{2+}$ was binding to TTA-HS $\mathrm{x}$ and mediating the reaction in the LLPS regions.

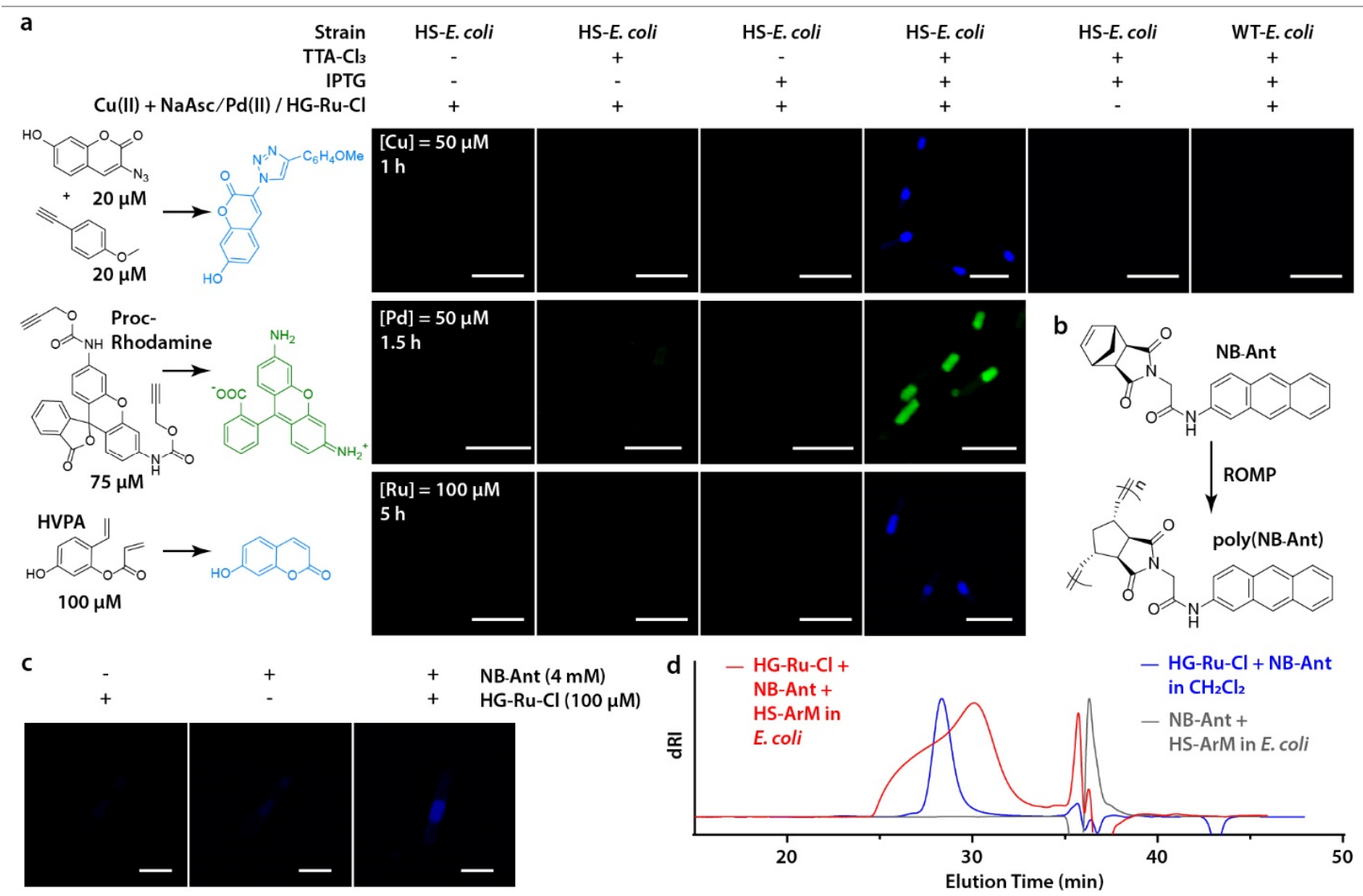

Figure 2. Intracellular reactions mediated by ArMs in catalytic compartments within E. coli. (a) Confocal microscopic images showing the ArM compartments' essentiality in mediating intracellular CuAAC, Procremoval and RCM reactions. (b) Schematic illustration of ROMP of an anthracene-norbornene (NB-Ant) monomer. (c) Confocal microscopic images showing the ROMP of the AIEgen monomer, NB-Ant, in E. coli. (d) GPC characterization of poly(NB-Ant)s obtained through intracellular polymerization and classic ROMP in dichloromethane.

In the above examples, metal ions were introduced and installed on the "empty" ligand located on the crosslinker TTA-Cl $\mathbf{3}_{\mathbf{3}}$. Yet, for many organometallic catalysts, it is impossible to simply mix a metal ion with ligands to afford the desired catalytic complex, therefore the corresponding cofactors may not be introduced into ArM systems in this way. Considering that it is too strenuous to prepare a crosslinker from each cofactor of interest, we devised a way to circumvent this issue. As we already knew that the dimerized or trimerized HS proteins were simply triggers for LLPS, we may still utilize monomeric HS to install more complex metal cofactors in the ArM compartments (Figure 1b, 1c). Thus, we prepared a chloroalkyl-functionalized Hoveyda-Grubbs catalyst, HG-Ru-Cl, and added this compound together with TTA-Cl $\mathbf{C}_{3}$ to HS-E. coli. Similarly, we observed the LLPS of HS protein, and the 
resulting Ru-HS-ArM compartments showed excellent activity toward 5-hydroxy-2-vinylphenyl acrylate (HVPA), effecting ring-closing metathesis (RCM) to yield a fluorescent hydroxycoumarin (Figure 2a, row 3 and Supplemental Figure 5d). Unlike the first and only report of in vivo metathesis using Streptavidin-based ArM, there was no need to place the Ru-HS-ArM in the "cleaner" periplasm. The compartments formed by HS protein perfectly served as shelters like periplasm did, protecting the ArMs from the poisoning by coordinating biomolecules such as glutathione (GSH). Free HG-Ru-Cl foreseeably showed no intracellular activity (Figure 2a, column 2/3) because of its sensitivity toward intracellular nucleophiles.

Compared to unimolecular deprotection and bimolecular coupling reactions, chain polymerizations are far more challenging in an intracellular setting, as the growth of polymer chains involves repetitive, multi-step coupling of monomers on reactive chain-ends, thus the successful formation of a polymer chain is highly dependent on the turnover number of the corresponding intracellular catalyst. Using in situ generated $\mathrm{Cu}^{\mathrm{I}}$ and alkylbromide as the initiating system, we successfully conducted the free radical polymerization of sodium vinylbenzenesulfonate (NaVBS, Supplemental Figure 7) in HS-E. coli, which was previously only possible with a light initiation system. ${ }^{32}$ The alkylbromide- $\mathrm{Cu}^{\mathrm{I}}$ initiation system allowed end-group labeling of the resulting polymer (Supplemental Figure 7a). Yet, we moved to a more demanding polymerization: ring-opening metathesis polymerization (ROMP). ${ }^{33}$ Owing to the sensitivity of the corresponding catalyst such as Grubbs catalysts and Hoveyda-Grubbs catalysts, in vivo metathesis was difficult and the sole reported case involved only intramolecular RCM reaction in bacteria periplasm. ${ }^{19}$ Thus, we attempted to polymerize an anthracene-functionalized exo-norbornenyl monomer (NB-Ant) with our compartmentalized HS-E. coli containing Ru-HS-ArMs, NB-Ant itself has very weak fluorescence in aqueous environment, but upon polymerization, poly(NB-Ant) shows prominent aggregation-induced emission (AIE). With a confocal microscope, we was able to observe the AIE fluorescence of poly(NB-Ant) in ArM compartments in E. coli (Figure 2e). To further verify the success of this polymerization, we conducted a largerscaled preparation using Ru-HS-ArM containing HS-E. coli as the whole-cell catalyst, and the polymer product was extracted from lysed cells and characterized by nuclear magnetic resonance (NMR) and gel permeation chromatography (GPC). Results indubitably demonstrated that NB-Ant was successfully polymerized, and the product had decent molecular weight (Figure 2f). These results indicated that the Ru-HS-ArMs in artificial compartments had high turnover numbers for norbornene derivatives.

Nature creates enclosed microenvironments not only for a protective effect, but also for the purpose of constructing cascade reactions and facilitating synthetic pathways by utilizing neighboring effect and high local concentration of reaction intermediates. As our ArM-in-compartment system offers great versatility in introducing different metal cofactors for different abiotic reactions, we were wondering if ArMs of different kind could be included in the same compartment to facilitate tandem reactions. To explore the possibility, we synthesized a new substrate, 5-azido-2vinylphenyl acrylate (AVPA), which has to undergo both CuAAC coupling with ethynylanisole and intramolecular RCM to become fluorescent (Figure 3a). By incubating HS-E. coli with $\mathrm{CuSO}_{4} / \mathrm{NaAsc}, \mathbf{H G}-\mathbf{R u}-\mathbf{C l}$ and TTA-Cl $\mathbf{3}_{3}$, we created both $\mathrm{Cu}-\mathrm{HS}-\mathrm{ArM}$ and Ru-HS-ArM in artificial compartments and screened for the best condition for 
this model tandem reaction. We observed that at a 7:1 ratio of $\mathbf{H G - R u - C l}$ and $\mathrm{CuSO}_{4}$, AVPA and ethynylanisole treated HS-E. coli showed strongest fluorescence, whereas control groups with only one type of catalyst showed no fluorescence (Figure 3b, 3c). Presumably, excess $\mathrm{Ru}^{\mathrm{III}}$ was needed because free copper ion could enter E. coli and compartments more efficiently meanwhile having lower environmental sensitivity than HG-Ru-Cl. Control studies, in which both types of catalyst were present in cells but only one type of catalyst was included in the compartments, showed the necessity of placing both catalysts under the same shelter, so that their performance could be preserved and the cascade reaction could be facilitated (Supplemental Figure 8).

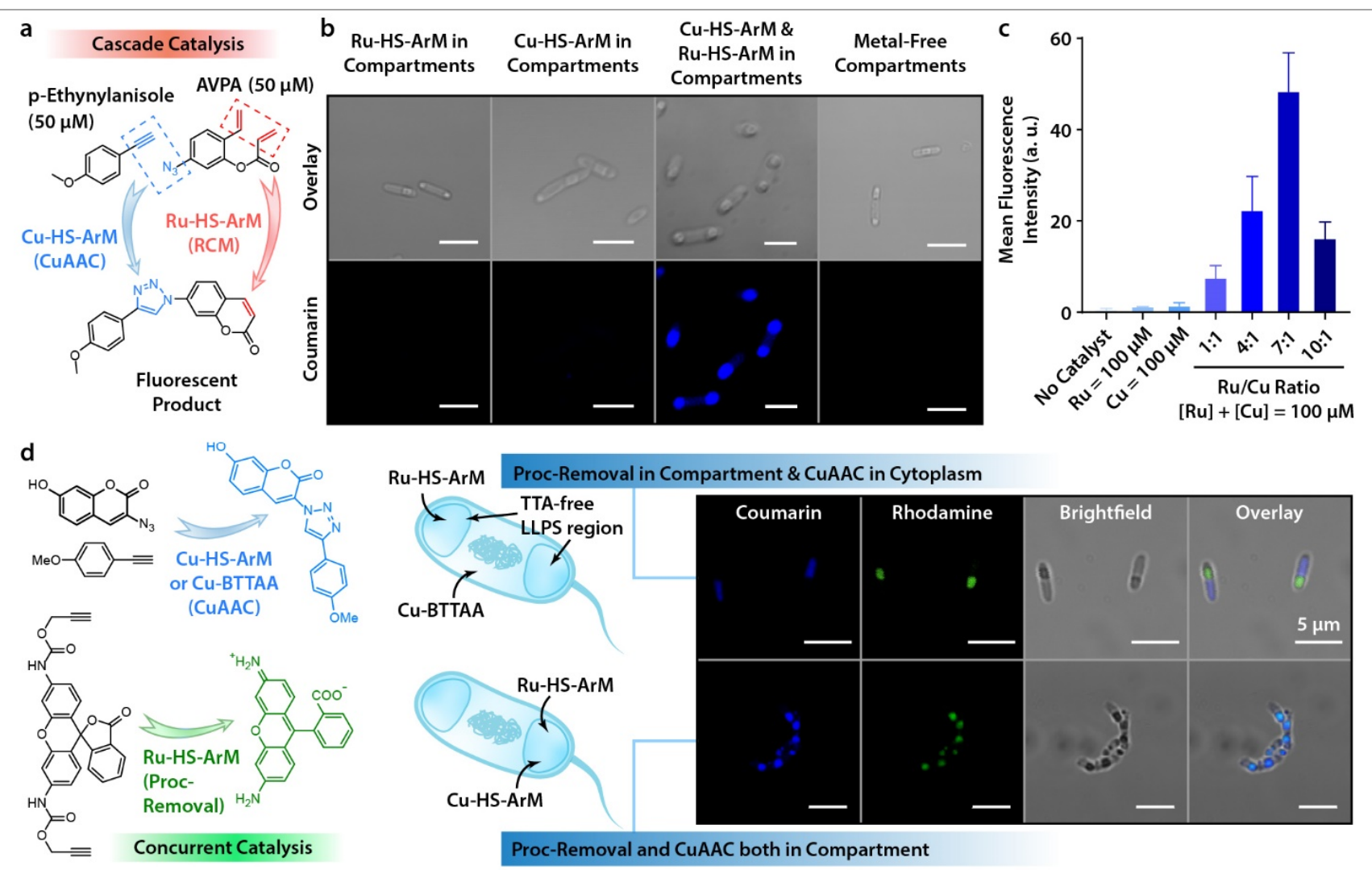

Figure 3. Cascade and concurrent catalysis mediated by E. coli containing catalytic compartments composed of ArMs. (a) Scheme of the fluorogenic cascade CuAAC-RCM reactions utilized in our study. (b) Confocal microscopic images indicating successful CuAAC-RCM cascade reaction taking place in ArM compartments containing copper and ruthenium cofactors. (c) The effect of metal cofactor ratio on the catalytic efficiency for the cascade reaction. (d) Schemes and confocal microscopic images of the two fluorogenic reactions that could be mediated concurrently and independently in compartmentalized $E$. coli. The two reactions could take place both in the compartments, or in compartments and cytoplasm respectively with physical separation.

Apart from cascade reactions, spatial separation of concurrent reactions can be an essential but difficult consideration for the construction of complex abiotic systems in cells. As we had observed the localization of catalyzed reactions in these compartmentalized ArM systems, it was reasonable to anticipate that such reactions in 
the ArM compartments could be spatially separated from the ones in the cytoplasm. To evaluate this hypothesis, we again constructed compartmentalized Ru-HS-E. coli using HG-Ru-Cl, HS-E. coli and $\mathbf{T r i s}_{\mathbf{3}} \mathbf{C l}_{\mathbf{3}}$. In addition to $\mathrm{RCM}$, this ruthenium ArM could also efficiently mediate the Proc-unmasking reactions similar to $\mathrm{Pd}^{\mathrm{II}}$ species. $\mathrm{Cu}$ BTTAA was utilized as the small molecular catalyst for conducting CuAAC in the cytoplasm, since the most active small molecular $\mathrm{Cu}$-TTA complexes were reported to retain a small portion of activity in cells and usable as an intracellular catalyst at high concentration. ${ }^{34,35}$ Because the LLPS were triggered using TTA-free Tris-Cl 3 , the compartments in this system were free of TTA ligands thus would not participate in CuAAC (Supplemental Figure 9a). When this E. coli system was incorporated by HG-Ru-ArM, and fed with ethynylanisole, azidocoumarin and Proc-Rhodamine together, spatially separated, concurrent catalysis of CuAAC and Proc-unmasking could be clearly observed under a confocal microscope, with the triazole-coumarin product appearing in the cytoplasm and free rhodamine in the ArM compartments (Figure 3d, top, and Supplemental Figure 9b, 9c). Not surprisingly, the concurrent reactions could also be both localized within the compartments when both ArMs were included in the LLPS regions. In this case, the triazole-coumarin and free rhodamine products were both observed in the compartments (Figure 3d, bottom, and Supplemental Figure 9b, 9c).

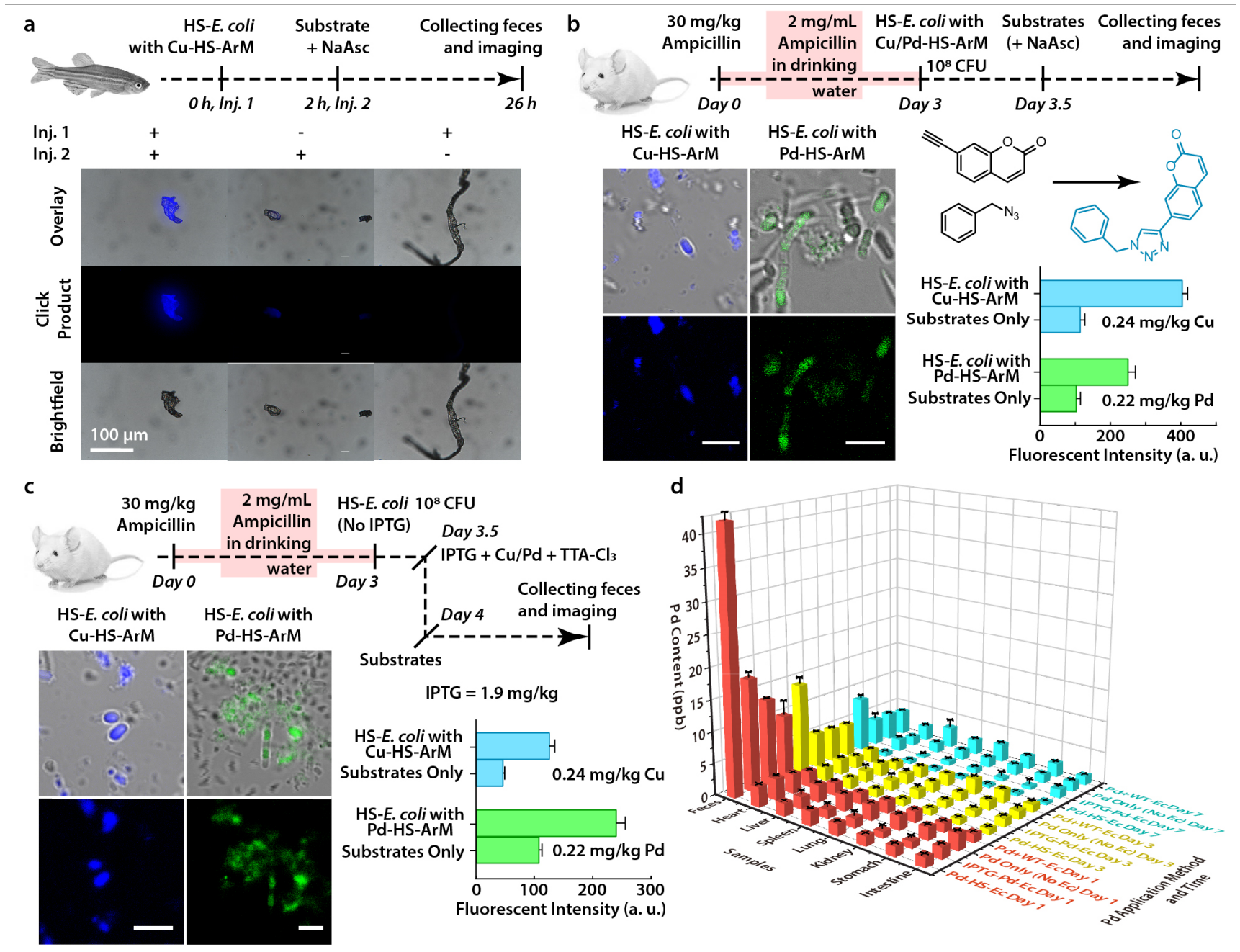


Figure 4. (a) Fluorescent images showing the successful coupling of ethynylanisole and azidocoumarin in zebrafish, mediated by ArM-LLPS-containing HS-E. coli as the whole-cell catalyst. (b) Confocal microscopic images showing successful CuAAC and Proc-Rhodamine unmasking reactions in mice intestine, mediated by ArM-LLPScontaining HS-E. coli as the whole-cell catalysts. (c) Confocal microscopic images showing that working wholecell catalysts based on HS-E. coli could be induced by the addition of IPTG and metal cofactors. (d) ICP-MS analysis of mice organs and feces after in vivo palladium-based catalysis was performed. A predominant portion of metal was removed from body through feces excretion. Pd-HS-Ec group: mice treated with pre-made whole-cell Pd catalyst, i.e., compartmentalized E. coli with Pd-HS-ArMs. IPTG-Pd-Ec group: mice treated with HS-E. coli and then Pd, IPTG and TTA- $\mathrm{Cl}_{3}$ to form compartmentalized whole-cell catalyst in intestine. Pd only group: mice treated with only $\mathrm{Pd}(\mathrm{OAc})_{2}$. $\mathrm{Pd}+\mathrm{WT}-\mathrm{Ec}$ group: mice treated with wild-type E. coli (no HS protein expression) and then Pd, IPTG and TTA-Cl $\mathbf{3}_{3}$.

One of the important goals for constructing ArMs that work in cells is to create whole-cell catalysts ${ }^{36}$ for abiotic transformations. The ArM-compartment system based on HS-E. coli is likely an excellent candidate for this purpose, because of the large volume of ArM compartments within bacteria cells and the versatility in cofactor introduction. Indeed, in a large scale (1 L) setting with compartmentalized HS-E. coli as whole catalysts, products from the ArMmediated reactions could be isolated from bacteria lysate (Supplemental Figure 10). The process could be viewed as a miniature abiotic fermentation procedure in which artificial cofactors were needed. One step forward, considering the low pathogenicity of E. coli, such bacteria may even serve as vessels to perform desired catalysis in live animals. As a proof of concept, we intraperitoneally injected compartmentalized, Cu-HS-ArM-containing HS- $E$. coli into zebrafish. After $2 \mathrm{~h}$, sodium ascorbate, azidocoumarin and ethynylanisole were introduced in the $2^{\text {nd }}$ injection, and fish feces were collected and imaged after another $1 \mathrm{~d}$. Observation of the collected feces clearly indicated successful CuAAC coupling in the fish, with strong fluorescence observable for the feces from the HSE. coli group (Figure 4a). No discernible fluorescence was seen for the control group. Obviously, HS-E. coli served as an abiotic whole-cell catalyst successfully in this case.

One possible advantage for conducting in vivo abiotic transformations in whole-cell catalyst is the localized catalyst - many of which are likely to be heavy metal complexes in nature and may lead to associated toxicity in animal hosts. Being "captured in cells", these heavy-metal-containing ArMs may be excreted together with the cells without leakage, minimizing possible impact that may be caused to the host by the administration of metal catalysts. $E$. coli, actually, is a good candidate cell for this purpose, because it can colonize the lower intestine of endotherms and get excreted with feces. Thus, with the reliable catalytic performance of our compartmentalized HS-E. coli systems, we proceeded to check their potential as localized, non-pathogenic, abiotic whole-cell catalysts with low risk of heavy metal poisoning. We adopted a reported method to colonize the intestine of live mice by intragastric gavage of HS-E. coli containing pre-established ArM-LLPS compartments (Figure 4b). After the colonization, the mice were fed with fluorogenic substrates (as well as sodium ascorbate for CuAAC), and their feces were collected and observed. Fluorescent imaging of the feces samples clearly indicated the presence of live HS-E. coli containing 
ArM-LLPS compartments, with the observation of unambiguous fluorescence from the triazole-coumarin and rhodamine products for $\mathrm{Cu}^{\mathrm{I}}$ - or $\mathrm{Pd}^{\mathrm{II}}$-catalyzed reactions (Supporting Video 1 and 2). This whole-cell catalyst could also be "prepared" in vivo. By colonizing compartment-free HS-E. coli in mice intestine first followed by feeding the mice with IPTG and metal cofactors, similar ArM-LLPS-containing whole-cell catalytic systems could form and show activity for CuAAC or Proc-unmasking reactions (Figure 4c). Very importantly, the whole-cell catalytic set-up shown above traps heavy metal species within bacterial cells may significantly reduce the risk of heavy metal poisoning by performing in vivo abiotic catalysis. Considering copper, as one of the trace essential elements, is regularly taken through food and can be excreted through natural metabolic pathways, ${ }^{37}$ potentially creating high background for the study, we used Pd-catalysis as a model to study the effect of this whole-cell system on heavy metal distribution in mice. By examining the metal content of feces and organs of the sacrificed mice after in vivo catalysis using inductively coupled plasma mass spectrometry (ICP-MS), the excretion and accumulation of metals could be evaluated. For mice treated with compartmentalized E. coli containing Pd-HS-ArM, highest content of Pd in the feces and lowest contents of Pd in the organs was observed, followed by mice treated with HS-E. coli activated by IPTG feeding (Figure 4d). Mice treated with wildtype E. coli plus Pd, or Pd only, showed lower metal contents in the feces and higher accumulation in the organs. As such, this ArM-based whole-cell approach may provide a solution for metal toxicity issues associated with in vivo abiotic catalysis.

\section{References}

1 Renata, H., Wang, Z. J. \& Arnold, F. H. Expanding the Enzyme Universe: Accessing Non-Natural Reactions by Mechanism-Guided Directed Evolution. Angew. Chem. Int. Ed. 54, 3351-3367 (2015).

2 Hyster, T. K. \& Ward, T. R. Genetic Optimization of Metalloenzymes: Enhancing Enzymes for NonNatural Reactions. Angew. Chem. Int. Ed. 55, 7344-7357 (2016).

3 Vornholt, T. et al. Systematic engineering of artificial metalloenzymes for new-to-nature reactions. Sci. Adv. 7, eabe4208 (2021).

4 Wilson, Y. M., Dürrenberger, M., Nogueira, E. S. \& Ward, T. R. Neutralizing the Detrimental Effect of Glutathione on Precious Metal Catalysts. J. Am. Chem. Soc. 136, 8928-8932 (2014).

5 Rottmann, P., Ward, T. \& Panke, S. Compartmentalization - A Prerequisite for Maintaining and Changing an Identity. Chimia 70, 428-431 (2016).

6 Alberti, S., Gladfelter, A. \& Mittag, T. Considerations and Challenges in Studying Liquid-Liquid Phase Separation and Biomolecular Condensates. Cell 176, 419-434 (2019).

7 Hyman, A. A., Weber, C. A. \& Jülicher, F. Liquid-Liquid Phase Separation in Biology. Annu. Rev. Cell Dev. Bi 30, 39-58 (2014).

8 Wei, S.-P. et al. Formation and functionalization of membraneless compartments in Escherichia coli. Nat. Chem. Biol. 16, 1143-1148 (2020).

9 Coelho Pedro, S., Brustad Eric, M., Kannan, A. \& Arnold Frances, H. Olefin Cyclopropanation via Carbene Transfer Catalyzed by Engineered Cytochrome P450 Enzymes. Science 339, 307-310 (2013). 
10 Song Woon, J. \& Tezcan, F. A. A designed supramolecular protein assembly with in vivo enzymatic activity. Science 346, 1525-1528 (2014).

11 Wilson, M. E. \& Whitesides, G. M. Conversion of a protein to a homogeneous asymmetric hydrogenation catalyst by site-specific modification with a diphosphinerhodium(I) moiety. J. Am. Chem. Soc. 100, 306307 (1978).

12 Yu, F. et al. Protein Design: Toward Functional Metalloenzymes. Chem. Rev. 114, 3495-3578 (2014).

13 Fischer, S., Ward, T. R. \& Liang, A. D. Engineering a Metathesis-Catalyzing Artificial Metalloenzyme Based on HaloTag. ACS Catal. 11, 6343-6347 (2021).

14 Key, H. M., Dydio, P., Clark, D. S. \& Hartwig, J. F. Abiological catalysis by artificial haem proteins containing noble metals in place of iron. Nature 534, 534-537 (2016).

15 Zastrow, M. L., Peacock, A. F. A., Stuckey, J. A. \& Pecoraro, V. L. Hydrolytic catalysis and structural stabilization in a designed metalloprotein. Nat. Chem. 4, 118-123 (2012).

16 Davis, H. J. \& Ward, T. R. Artificial Metalloenzymes: Challenges and Opportunities. ACS Cent. Sci. 5, 1120-1136 (2019).

17 Schwizer, F. et al. Artificial Metalloenzymes: Reaction Scope and Optimization Strategies. Chem. Rev. 118, 142-231 (2018).

18 Creus, M. et al. X-Ray Structure and Designed Evolution of an Artificial Transfer Hydrogenase. Angew. Chem. Int. Ed. 47, 1400-1404 (2008).

19 Jeschek, M. et al. Directed evolution of artificial metalloenzymes for in vivo metathesis. Nature 537, 661665 (2016).

20 Zhao, J. et al. Genetic Engineering of an Artificial Metalloenzyme for Transfer Hydrogenation of a SelfImmolative Substrate in Escherichia coli's Periplasm. J. Am. Chem. Soc. 140, 13171-13175 (2018).

21 Jeschek, M., Panke, S. \& Ward, T. R. in Method. Enzymol. Vol. 580 (ed Vincent L. Pecoraro) 539-556 (Academic Press, 2016).

22 Garabedian, M. V. et al. Designer membraneless organelles sequester native factors for control of cell behavior. Nat. Chem. Biol. 17, 998-1007 (2021).

23 Gomes, E. \& Shorter, J. The molecular language of membraneless organelles. J. Biol. Chem. 294, 71157127 (2019).

24 Boeynaems, S. et al. Protein Phase Separation: A New Phase in Cell Biology. Trends Cell Biol. 28, 420435 (2018).

25 Liu, Y. et al. AgHalo: A Facile Fluorogenic Sensor to Detect Drug-Induced Proteome Stress. Angew. Chem. Int. Ed. 56, 8672-8676 (2017).

26 Lin, Y., Protter, David S. W., Rosen, Michael K. \& Parker, R. Formation and Maturation of PhaseSeparated Liquid Droplets by RNA-Binding Proteins. Mol. Cell 60, 208-219 (2015).

27 Nott, Timothy J. et al. Phase Transition of a Disordered Nuage Protein Generates Environmentally Responsive Membraneless Organelles. Mol. Cell 57, 936-947 (2015). 

in Live Cells Using Solvatochromic and Molecular Rotor-Based Fluorophores. Biochemistry 57, 46634674 (2018).

29 Fares, M. et al. A Molecular Rotor-Based Halo-Tag Ligand Enables a Fluorogenic Proteome Stress Sensor to Detect Protein Misfolding in Mildly Stressed Proteome. Bioconjugate Chem. 29, 215-224 (2018).

30 Jung, K. H., Kim, S. F., Liu, Y. \& Zhang, X. A Fluorogenic AggTag Method Based on Halo- and SNAPTags to Simultaneously Detect Aggregation of Two Proteins in Live Cells. ChemBioChem 20, 1078-1087 (2019).

31 Ellis, R. J. Macromolecular crowding: an important but neglected aspect of the intracellular environment. Curr. Opin. Struc. Biol 11, 114-119 (2001).

32 Geng, J. et al. Radical polymerization inside living cells. Nat. Chem. 11, 578-586 (2019).

33 Bielawski, C. W. \& Grubbs, R. H. Living ring-opening metathesis polymerization. Prog. Polym. Sci. 32, $1-29$ (2007).

34 Uttamapinant, C. et al. Fast, Cell-Compatible Click Chemistry with Copper-Chelating Azides for Biomolecular Labeling. Angew. Chem. Int. Ed. 51, 5852-5856 (2012).

35 Yang, M. et al. Biocompatible click chemistry enabled compartment-specific pH measurement inside E. coli. Nat. Commun. 5, 4981 (2014).

36 Klibanov Alexander, M. Immobilized Enzymes and Cells as Practical Catalysts. Science 219, 722-727 (1983).

37 Camakaris, J., Voskoboinik, I. \& Mercer, J. F. Molecular Mechanisms of Copper Homeostasis. Biochem. Bioph. Res. Co. 261, 225-232 (1999). 


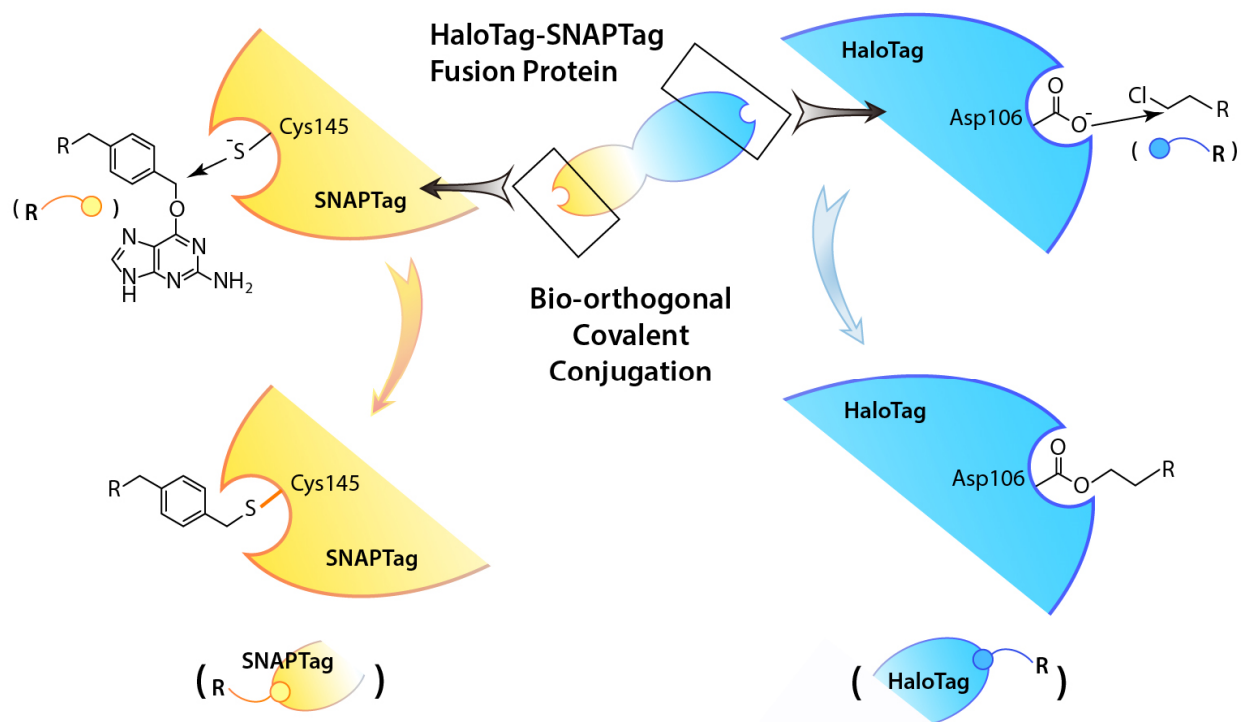

Supplemental Figure 1. Bio-orthogonal coupling reactions utilized in HS-ArM assembly and labeling.

HaloTag is a modified haloalkane dehalogenase that can have its Asp106 residue irreversibly displace the chloride on a chloroalkyl substrate. SNAPTag is a truncated and modified human $O^{6}$-alkylguanine-DNA alkyltransferase that can react with a $O^{6}$-benzylguanine substrate with its Cys 145 residue, by displacing the $O^{6}$ benzylguaninyl moiety. The two coupling reactions are both bio-orthogonal and efficient. 

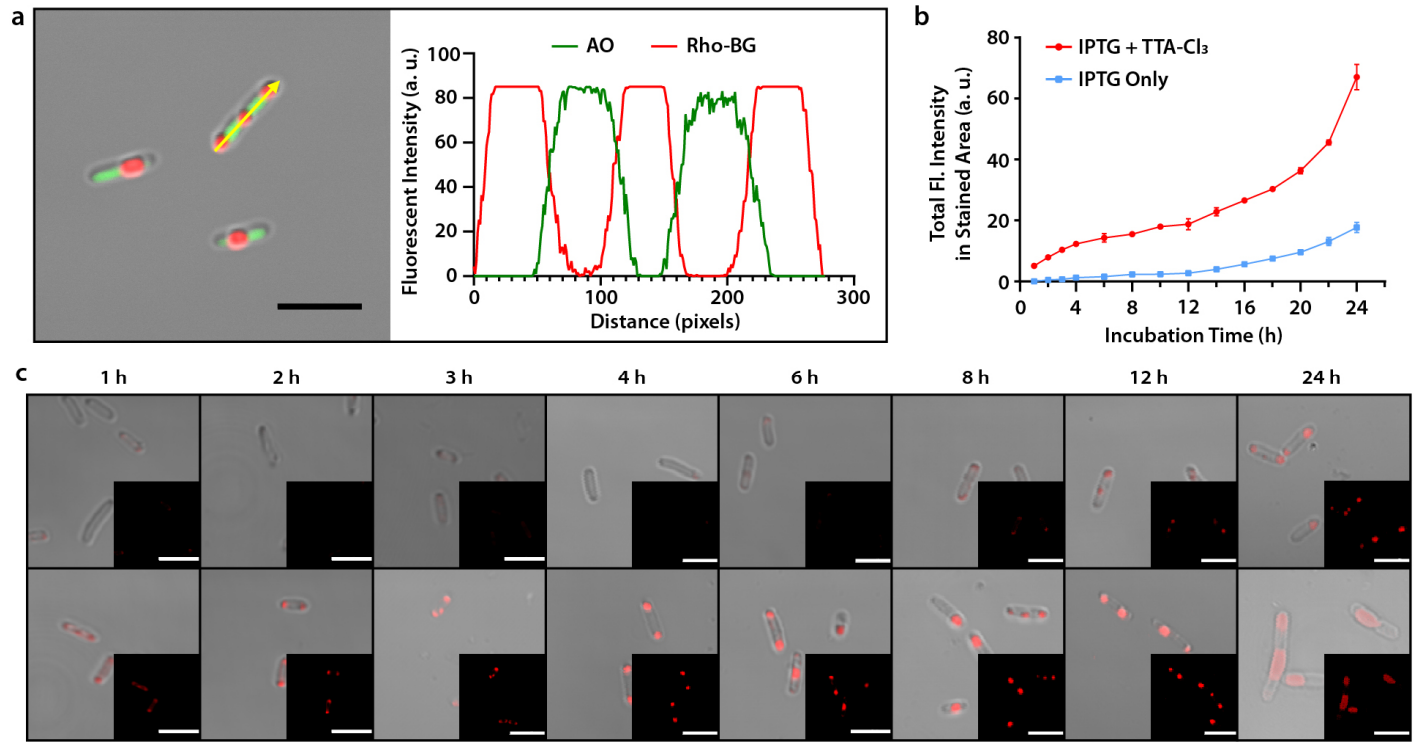

Supplemental Figure 2. Studies on HS-E. coli's compartmentalization process by confocal microscopy.

(a) Fluorescent image analysis of compartmentalized E. coli stained with acridine orange (AO) and Rho-BG. AO stains bacterial DNA and Rho-BG stains HaloTag-ArM containing compartments. The curve figure on the right denotes the fluorescent intensity of pixels along the line indicated with the yellow arrow in the left figure.

(b) Kinetics of the formation of LLPS compartments in HS-E. coli with or without the presence of IPTG. (c) Confocal microscopic images of HS-E. coli stained with Rho-BG after the addition of IPTG and fluorophore. Bacteria that were not treated with IPTG served as the control. Scale bar $=5 \mu \mathrm{m}$. 

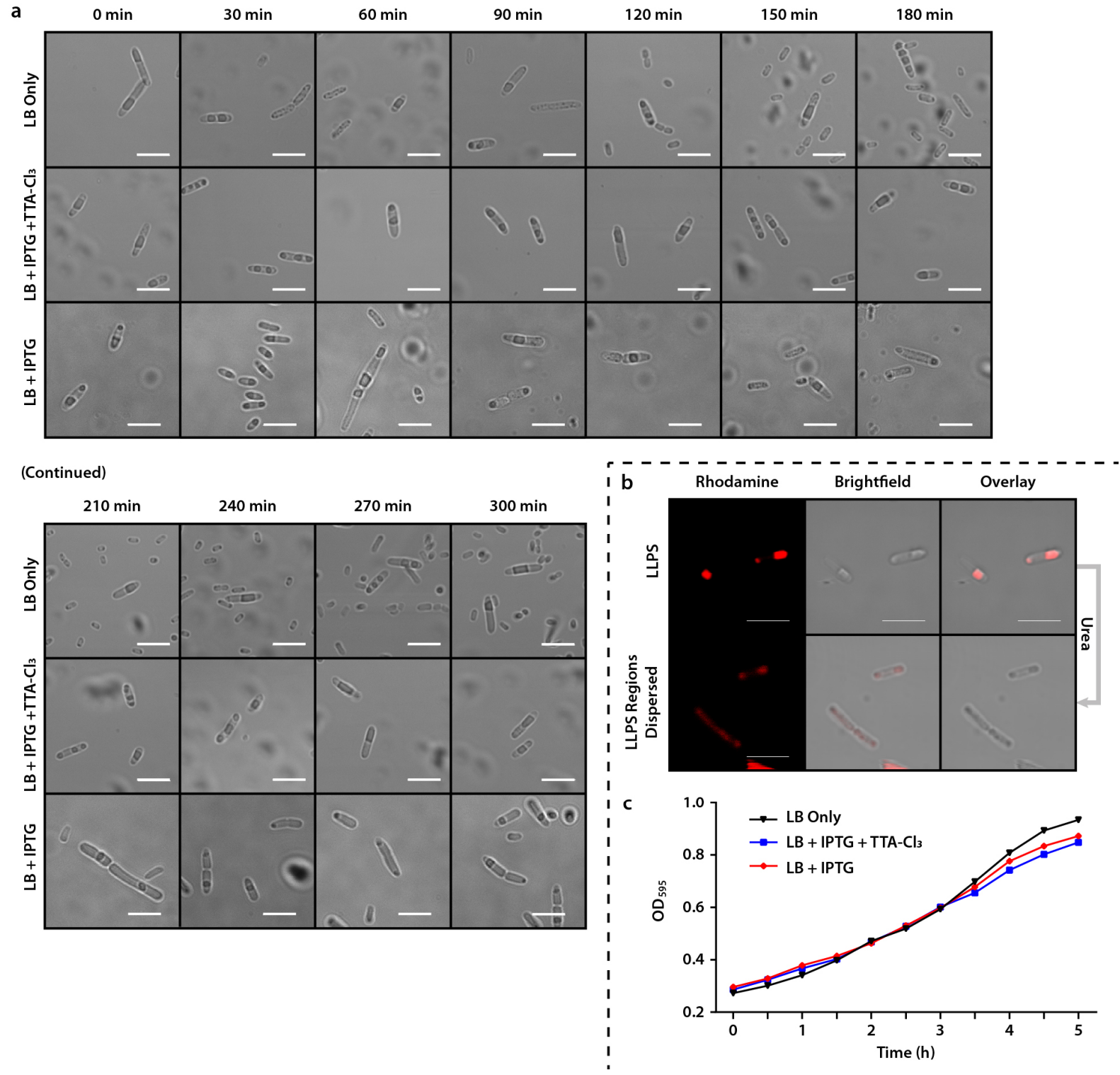

\section{Supplemental Figure 3. Reproduction and compartmentalization of the compartmentalized HS-E. coli.}

(a) Confocal microscopic images of the "offspring" of the compartmentalized HS-E. coli. These bacterial cells, after several cell divisions, still showed apparent compartmentalization within the cells. Bacteria cells can inherit some of the aggregated HS proteins after each cell division, thus no more crosslinkers ( to initiate the LLPS process. IPTG is still needed to produce more HS protein for the descendants.

(b) Disruption of the LLPS phase (labeled with Rho-BG) by the treatment of urea, a well-known hydrogen bonding disruptor and protein denaturing reagent. (c) Growth of compartmentalized HS-E. coli in different conditions, monitored using optical density measurements. IPTG and TTA-Cl $\mathrm{C}_{3}$ did not show significant impact on bacterial growth. 

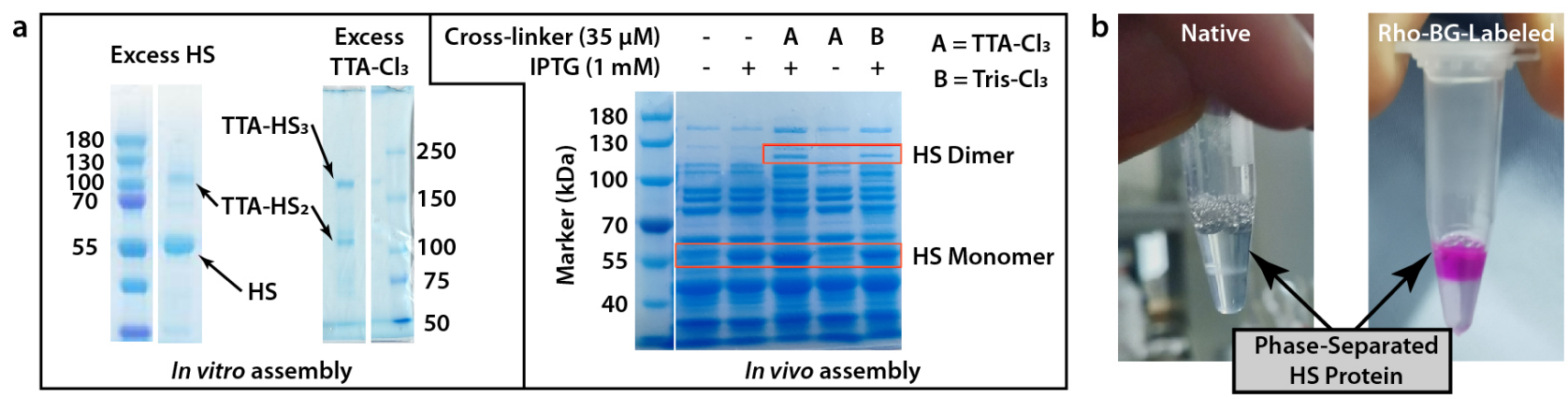

Supplemental Figure 4. In vitro and in vivo multimerization and phase separation of HS protein triggered by TTA-Cl $\mathbf{H}_{3}$.

(a) Formation of $\mathrm{HS}$ dimer and trimer in vivo and in vitro with the addition of TTA-Cl 3 , as revealed by gel analysis.

(b) Photographs of phase-separated HS protein with the addition of $\mathrm{TTA}-\mathrm{Cl}_{3}$ in dextran-70 solution. In the right photo, HS was labeled with Rho-BG (magenta) for clearer view. 
a

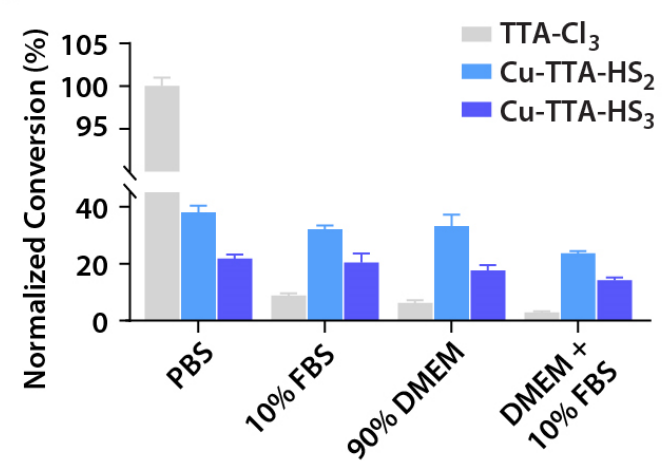

b

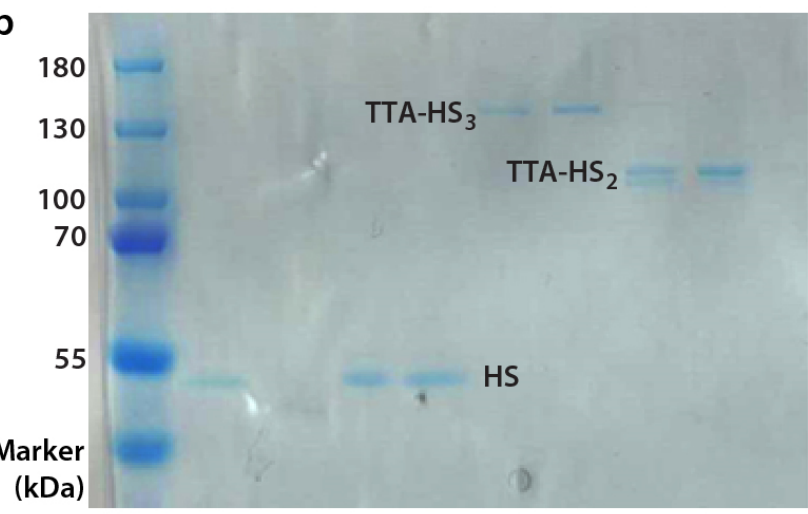

Supplemental Figure 5. Catalytic efficiency evaluation of the copper complexes: small molecular Cu-TTA$\mathrm{Cl}_{3}$ and multimeric HS protein bearing Cu-TTA moiety (Cu-TTA-HS 2 and Cu-TTA-HS $)_{3}$.

(a) Comparison on catalytic capabilities (as represented by substrate conversion in a set time) of $\mathrm{Cu}^{\mathrm{I}}-\mathrm{HS}_{3}, \mathrm{Cu}^{\mathrm{I}}-\mathrm{HS}_{2}$, $\mathrm{Cu}^{\mathrm{I}}-\mathrm{TTA}-\mathrm{Cl}_{3}$ in biologically relevant media. While the small molecular complex, Cu-TTA-Cl${ }_{3}$, showed significantly higher activity compared to the multimeric ArM, presumably because of low steric hindrance from the coordination sphere, its activity was drastically reduced by biomolecules in FBS and DMEM. In contrast, the activities of ArMs were not quite affected by complex environment. More hindered ArM (Cu-HS $)_{3}$ showed higher environment resistance and lower innate activity. Substrate: azidocoumarin and ethynylanisole $(20 \mu \mathrm{M} / 40 \mu \mathrm{M})$, $[\mathrm{Cu}]=100 \mu \mathrm{M}, 60 \mathrm{~min}$.

(b) Gel image of purified HS, HS dimer and HS trimer. The dimer and trimer were from the assembly of HS and TTA- $\mathrm{Cl}_{3}$ and was used as the protein scaffold for ArMs used in the study in panel (a) of this data figure. 

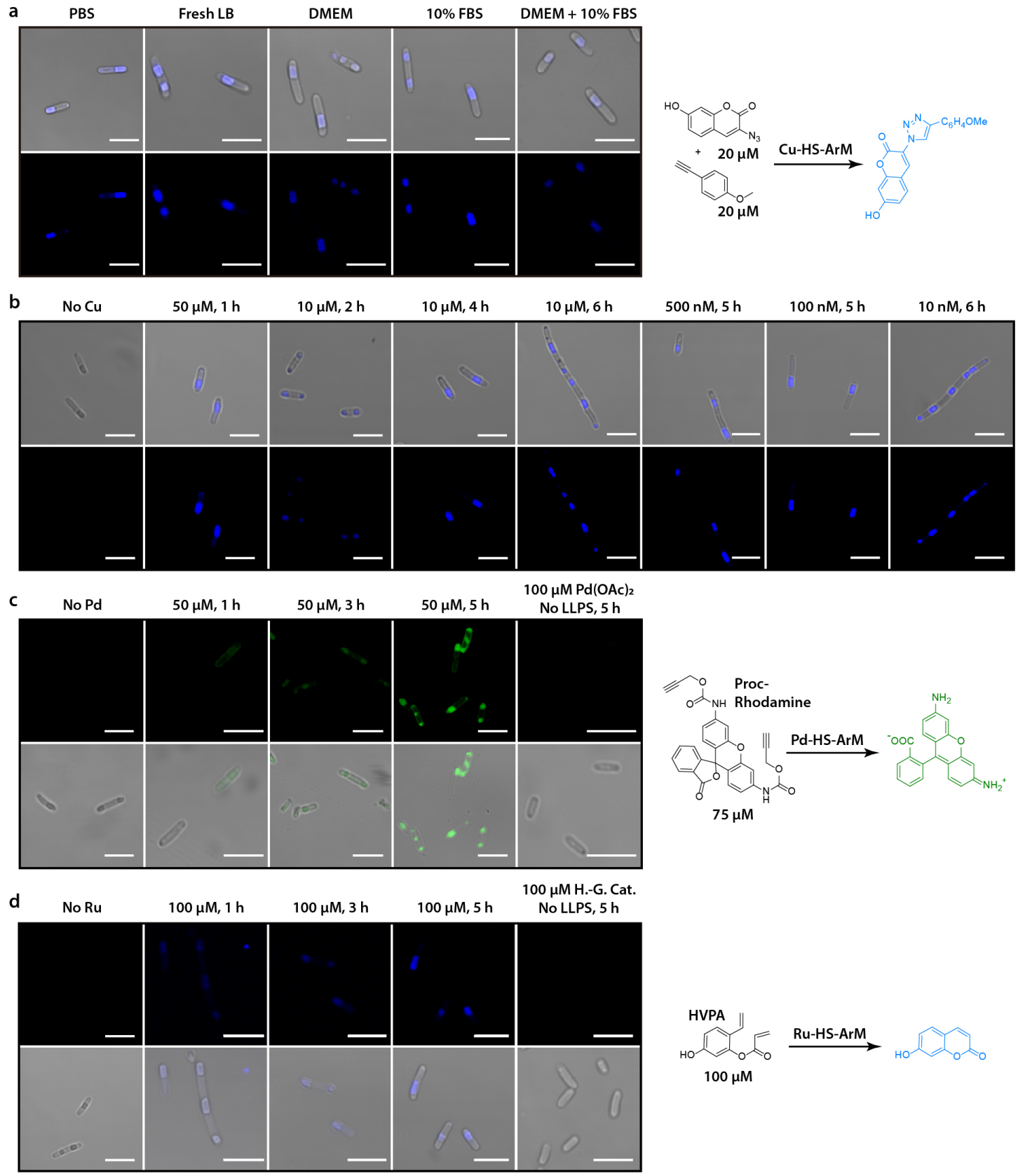

Supplemental Figure 6. Catalytic efficiency evaluation and comparison of compartmentalized HS-E. coli in different media with different reaction conditions.

(a) Confocal microscopic images showing successful CuAAC catalysis in compartmentalized HS-E. coli in different media. For experiments using media containing FBS, the media were briefly switched to $\mathrm{CuSO}_{4}$ containing FBS during copper incubation.

(b-d) Visualization of intracellular CuAAC, Proc-deprotection and RCM in HS-E. coli at different metal concentrations in different time periods. Scale bar $=5 \mu \mathrm{m}$. 


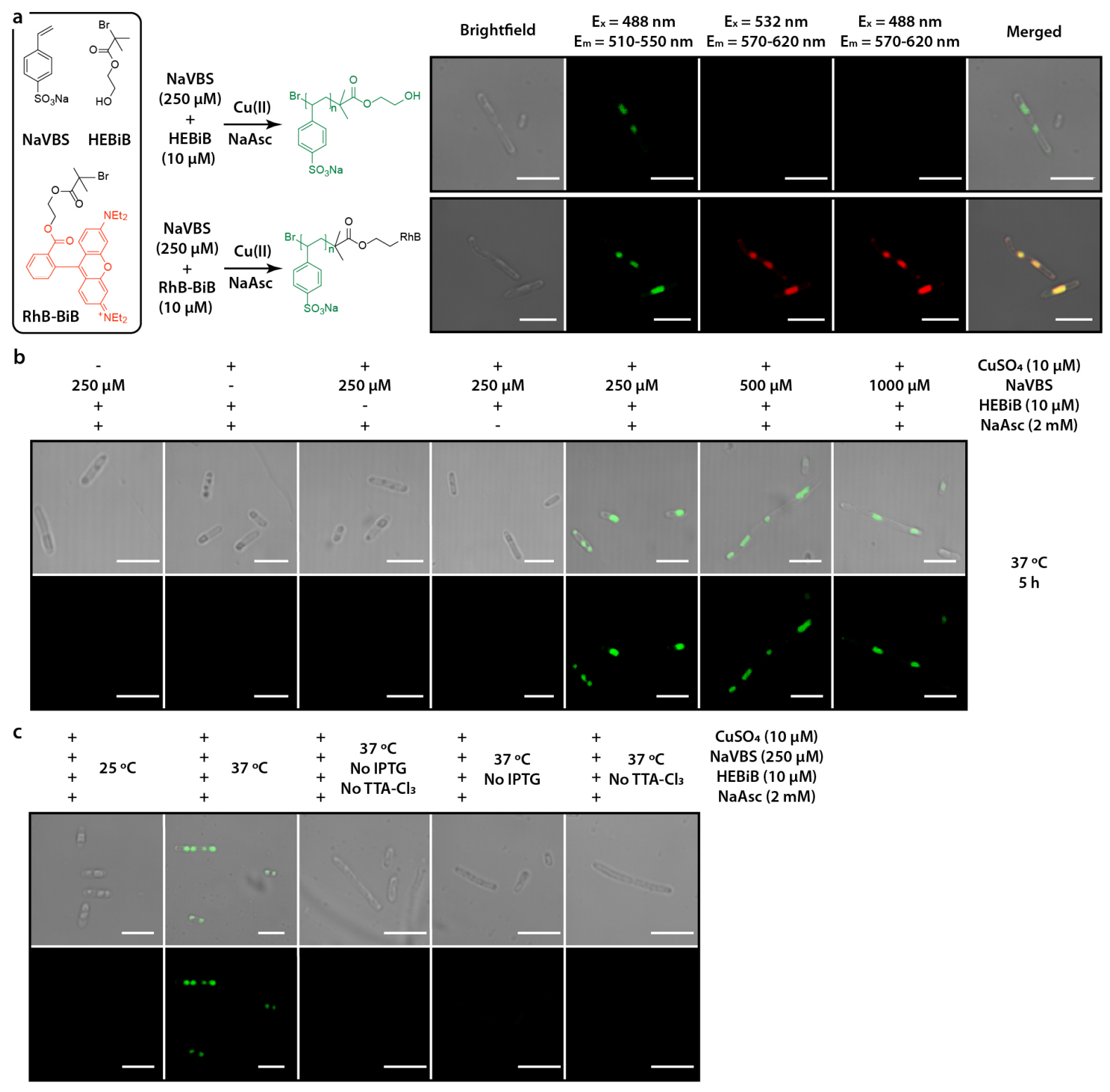

Supplemental Figure 7. Free radical polymerizations in compartmentalized HS-E. coli.

(a) Fluorogenic free radical polymerization of sodium benzenesulfonate (NaVBS) mediated by $\mathrm{Cu}-\mathrm{HS}-\mathrm{ArM}$ in compartmentalized HS-E. coli, using hydroxyethyl bromoisobutyrate (HEBiB) or rhodamine-functionalized bromoisobutyrate (RhB-BiB) as the initiator. FRET between rhodamine and poly(NaVBS) could be observed when RhB-BiB was used.

(b) Confocal microscopic study of intracellular NaVBS polymerization performed with different reagent concentrations.

(c) Confocal microscopic study of intracellular NaVBS polymerization performed in different control conditions. Slightly elevated temperature and LLPS compartmentalization are necessary for successful polymerization. 


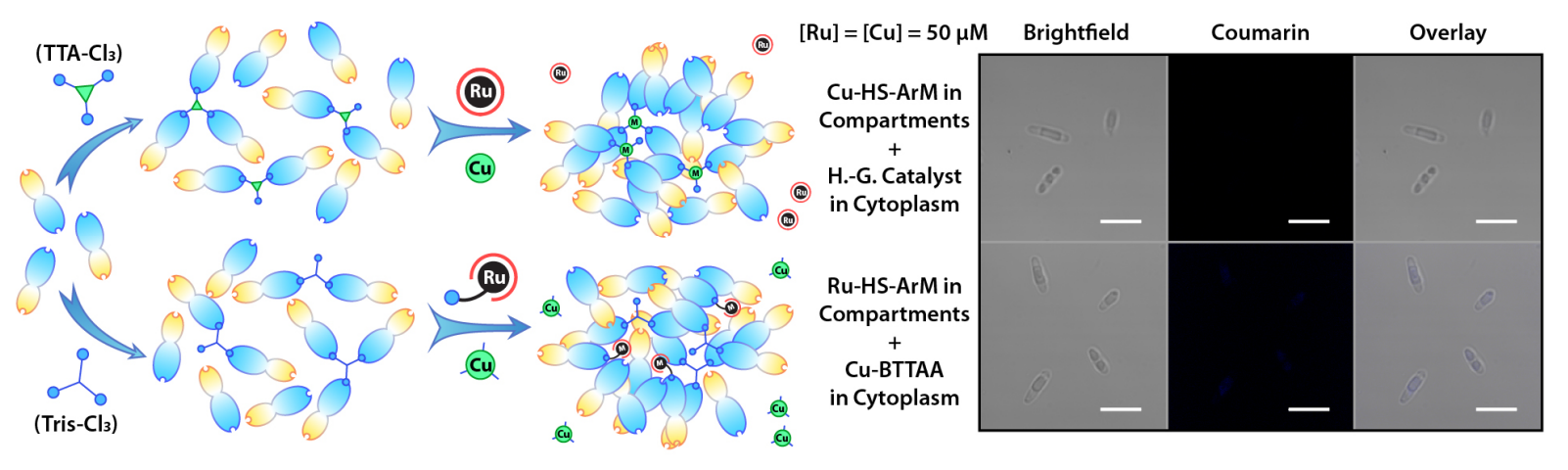

Supplemental Figure 8. Cartoon illustration of the establishment of catalytic systems with two catalytic species respectively in $\mathrm{HS}$ compartments and cytoplasm. By replacing $\mathrm{HG}-\mathrm{Ru}-\mathrm{Cl}$ with the native HoveydaGrubbs catalyst in the above cascade reaction system, HS-E. coli with in-compartment CuAAC catalyst and incytoplasm metathesis catalyst was established using TTA-Cl 3 (top). Similarly, by changing the crosslinker to a TTA-free crosslinker, Tris-Cl $\mathrm{C}_{3}, \mathrm{HG}-\mathrm{Ru}-\mathrm{Cl}$ could still be installed in $\mathrm{HS}$ compartments to form Ru-HS-ArMs, and the usage of small molecular Cu-BTTAA would generate a system with in-compartment metathesis catalyst and in-cytoplasm CuAAC catalyst (bottom). Both systems exhibited much weaker fluorescence after substrate incubation under confocal microscope. It can be clearly seen that the LLPS compartments are essential for successful cascade reaction catalysis. The "Ru in compartments $+\mathrm{Cu}$ in cytoplasm" group showed trace fluorescent signal because Cu-BTTAA was reported to retain a portion of activity in cells, and the RCMed intermediate could slowly diffuse out of the compartment and be catalyzed by Cu-BTTAA. 


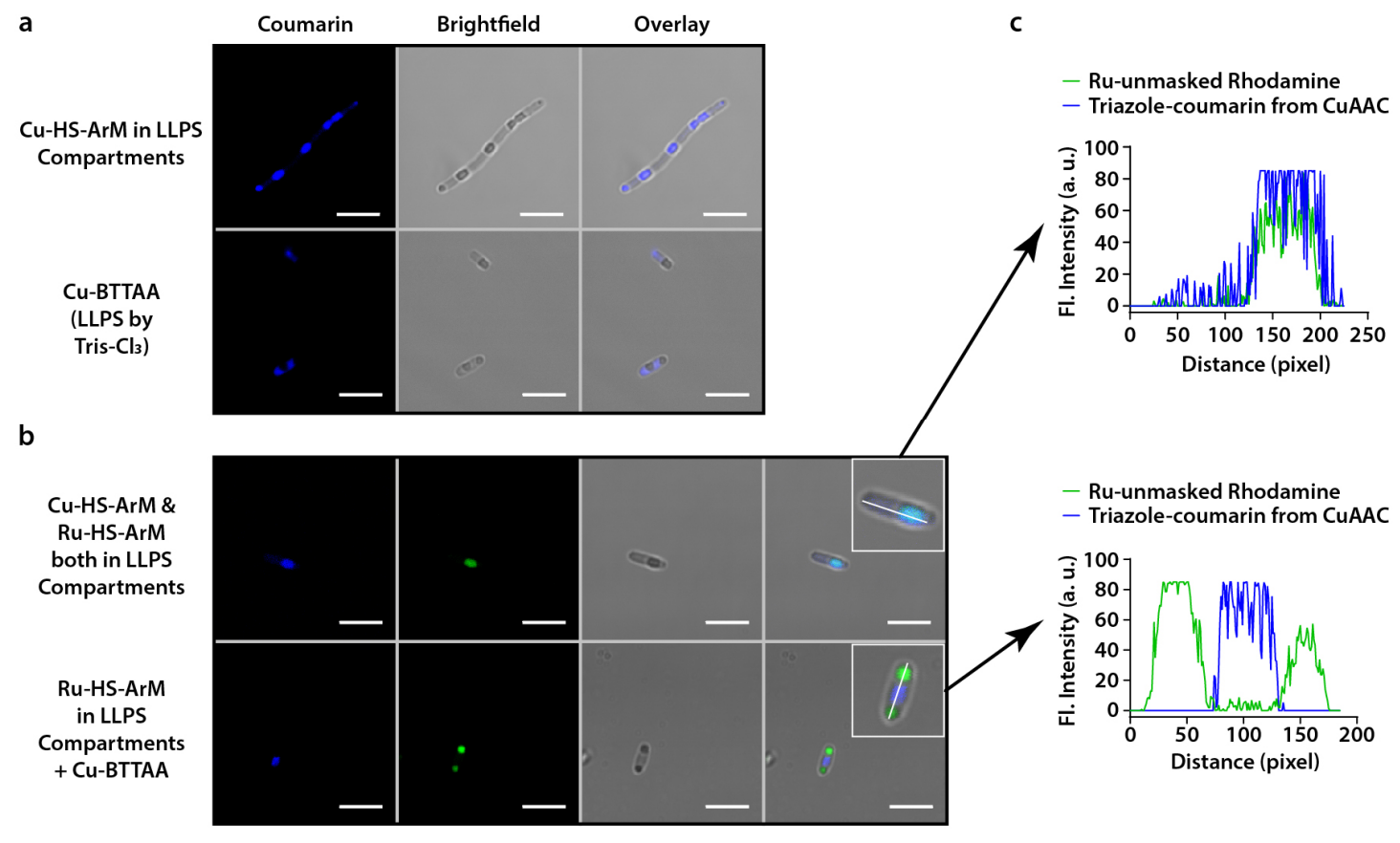

\section{Supplemental Figure 9. Spatial control of abiotic catalysis in compartmentalized HS-E. coli.}

(a) Confocal microscopic images showing the in-compartment catalysis by $\mathrm{Cu}-\mathrm{HS}-\mathrm{ArMs}$ in the LLPS regions, and in-cytoplasm catalysis by Cu-BTTAA when LLPS compartments were present and created by TTA-free crosslinker, Tris- $\mathrm{Cl}_{3}$.

(b) Confocal microscopic images showing the concurrent abiotic catalysis, namely $\mathrm{CuAAC}$ and rutheniummediated Proc-unmasking reaction, with spatial control. The two reactions could be mediated at the same time with spatial separation, or both in LLPS compartments.

(c) Pixel analysis of the fluorescent images, indicating the products from the two abiotic reactions could be spatially separated or be placed both in the compartments. 


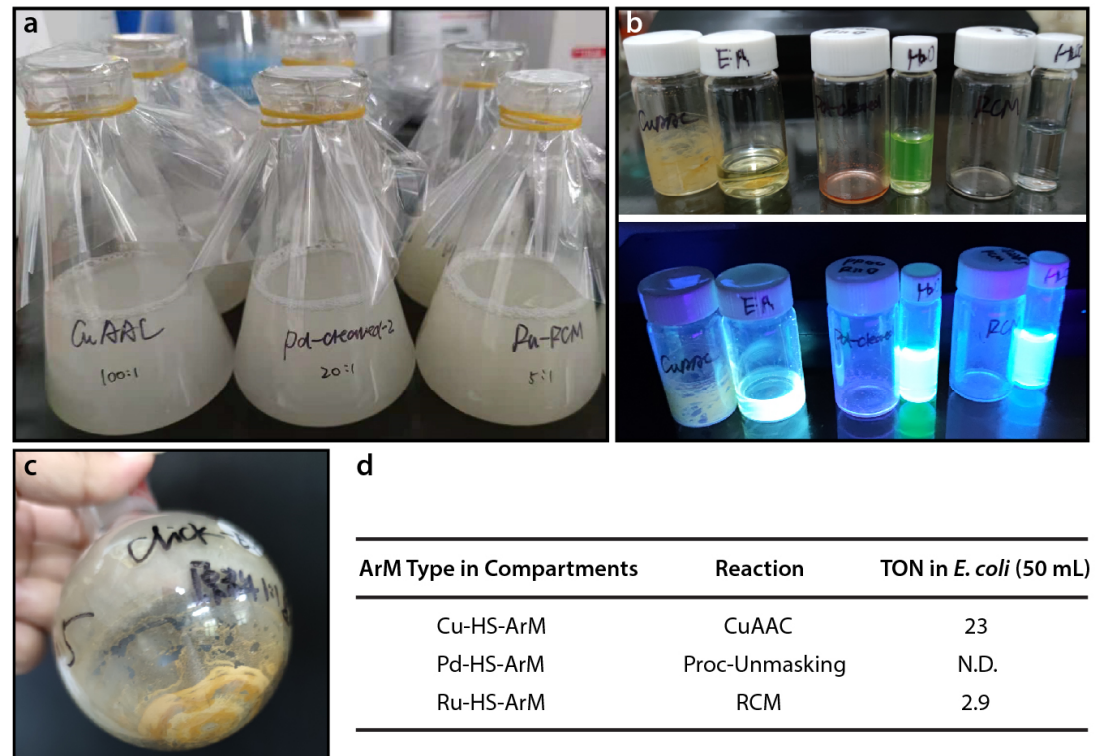

Supplemental Figure 10. Miniature abiotic "fermentation" by copper-, palladium- or ruthenium-containing whole-cell catalyst based on the compartmentalized HS-E. coli system.

(a) Photographs showing large scale molecular transformation $(200 \mathrm{~mL})$ using compartmentalized HS-E. coli with Cu-, Pd- or Ru-ArMs as whole-cell catalysts.

(b) Extracted CuAAC product (triazole-coumarin), Proc-removed rhodamine, and RCM product (hydroxycoumarin) in solid form and solution form. See Figure 2 for details of the catalytic transformations. These products were from whole-cell catalyzed reactions at 200-mL scale.

(c) Extracted CuAAC product (triazole-coumarin) from a whole-cell catalyzed CuAAC at 1-liter scale.

(d) Calculated turnover numbers of ArMs in E. coli's LLPS compartments for reactions conducted at 50-mL scale. The numbers were calculated based on the measured concentrations of intracellular metal species (by ICP-MS, [Cu] $=99 \mathrm{ppb}, 77 \%$ of added copper in total; $[\mathrm{Pd}]=85 \mathrm{ppb}, 71 \%$ of added palladium in total; $[\mathrm{Ru}]=110 \mathrm{ppb}, 86 \%$ of added ruthenium in total). N.D = not determined. 